\title{
Una nueva especie de Teagueia (Orchidaceae: Pleurothallidinae) del norte del Perú
}

\section{A new species of Teagueia (Orchidaceae: Pleurothallidinae) from Northern of Peru}

\author{
Miguel Chocce ${ }^{1}$, Nanette Vega $^{2}$, Margoth Acuña-Tarazona ${ }^{1}$, Jorge Arnaiz ${ }^{3}$ y Betty \\ Millán ${ }^{1}$ \\ 1 Departamento de Gimnospermas y Monocotiledóneas del Museo de Historia Natural de la Universidad Nacional Mayor de San Marcos. \\ Av. Arenales 1256, Apartado 14-0434, Lima 14, Perú. \\ 2 Departamento de Florística del Museo de Historia Natural de la Universidad Nacional Mayor de San Marcos, Lima - Perú. \\ 3 Laboratorio de Estudios en Biodiversidad. Facultad de Ciencias y Filosofía, Universidad Peruana Cayetano Heredia, Earth \& Environmental \\ Division, Amec (Perú) S.A. Lima - Perú \\ E-mail Miguel Chocce: mchocce@gmail.com, E-mail Nanette Vega: naveve2004@gmail.com, \\ E-mail Margoth Acuña-Tarazona: mareli.laura@gmail.com, E-mail Jorge Arnaiz: jorge.arnaiz@leb-upch.org, \\ E-mail Betty Millán: bmillans@unmsm.edu.pe
}

\section{Resumen}

Se describe e ilustra una nueva especie perteneciente al género Teagueia (Luer) Luer (Orchidaceae: Pleurothallidinae), proveniente de la región Lambayeque, al norte del Perú. Esta nueva especie es el primer reporte del género Teagueia (Luer) Luer para el Perú.

Palabras clave: Orchidaceae, Pleurothallidinae, Teagueia, nueva especie, Perú

\section{Abstract}

A new species of Teagueia (Luer) Luer (Orchidaceae: Pleurothallidinae) from the highlands of Northern Peru is described and illustrated with a black and white drawing. This species is the first record of genus Teagueia (Luer) Luer in Peru.

Keywords: Orchidaceae, Pleurothallidinae, Teagueia, new species, Peru

\section{Introducción}

El género Teagueia (Luer) Luer inicialmente fue propuesto por Luer (1986) como subgénero dentro del género Platystele. Las tres especies que conforman este grupo se caracterizan por las grandes flores con caudas sepalinas, el labio trilobulado con los lóbulos basales rodeando la columna y estigma bilobulado.

Luer (1990) en su monografía de Platystele, mantiene el subgénero Teagueia, pero incorpora una especie más al grupo. Posteriormente, Luer (1991) propuso elevar el rango de este grupo a género debido a características (venación de sépalos, y forma y tamaño de sépalos, pétalos y labio) que hacían inconsistente a este grupo con Platystele, hasta ese entonces el grupo contaba con seis especies, las cuales presentan poblaciones aisladas en los bosques que se encuentran entre los 2200 y 3000 m de elevación en los Andes de Colombia y Ecuador.

Jost (2004) reportó haber encontrado 26 nuevas especies (hasta el momento solamente han sido descritas cuatro especies) en cuatro cerros de la cuenca alta del río Pastaza (Ecuador, sugiriendo que tienen un reciente origen y provienen de un antepasado común, debido a su limitada distribución y a los caracteres compartidos entre ellas que son inusuales o ausentes en las seis especies de Teagueia previamente conocidas.

En el presente estudio se describe e ilustra Teagueia moisesii Chocce \& Acuña-Tarazona, el primer registro de este género para el Perú. El espécimen tipo proviene de los bosques montanos que se encuentran en el distrito de Cañaris, la provincia de Ferreñafe en la región Lambayeque, en el Norte del Perú.

\section{Teagueia moisesii Chocce \& Acuña-Tarazona sp.} nov.

Tipo: PERÚ: Lambayeque: Cañaris: Bosque montano, 3418 m de elevación, 23 de Mayo de 2008, M. Chocce, N. Vega, J. Arnaiz, S. Novoa, T. Huamán y E. Lucero 3894 (Holotipo: USM).

Similaris Teagueia phasmida (Luer \& Escobar) Luer, sed cum floribus minoribus, folia et ramicaulis maximis.

Hierba epífita de $12 \mathrm{~cm}$, simpodial, cortamente repente, rizoma de 0,5-1 cm de largo entre ramicaules. Raíces basales, delgadas, carnosas, blancas, $2 \mathrm{~mm}$ de grosor. Ramicaules delgados, $2 \mathrm{~cm}$ de largo, cubierta por tres vainas delgadas e imbricadas. Hojas erectas, delgadamente coriáceas, peciolado, 5,5-7 cm de largo (incluyendo peciolo), la lamina elíptica, obtusa, 3,5-4 x 1,2-1,5 cm, cuneada en la base. Inflorescencia racemosa, suberecta, de floración simultánea, hasta de $11 \mathrm{~cm}$; pedúnculo delgado, hasta $3,8 \mathrm{~cm}$; brácteas florales delgadas, $2 \mathrm{~mm}$ de largo; pedicelos de 2,5 mm de largo, ovario de 1,5 mm de largo; sépalos pubescentes, carinados, revolutos, sépalo dorsal púrpura, angostamente triangular, con el ápice largamente atenuado, 12 $\mathrm{mm}$ de largo, $2 \mathrm{~mm}$ de ancho (extendido), 3-nervado, los sépalos laterales similares, 12,5 mm de largo, $4 \mathrm{~mm}$ de ancho (extendido), unido $3 \mathrm{~mm}$ desde la base; pétalos púrpura, revolutos, cortamente pubescentes, triangulares, agudos, $2 \mathrm{~mm}$ de largo, $1 \mathrm{~mm}$ de ancho; labelo púrpura, poco pubescente, obovado, 3 $\mathrm{mm}$ de largo, 1,5 de ancho, ápice redondeado y cóncavo, con márgenes involutas hacia el centro del disco, disco con un callo elíptico, ligeramente prominente, lóbulos basales cortamente pubescentes en sus márgenes, angostamente obtuso, abrazando 


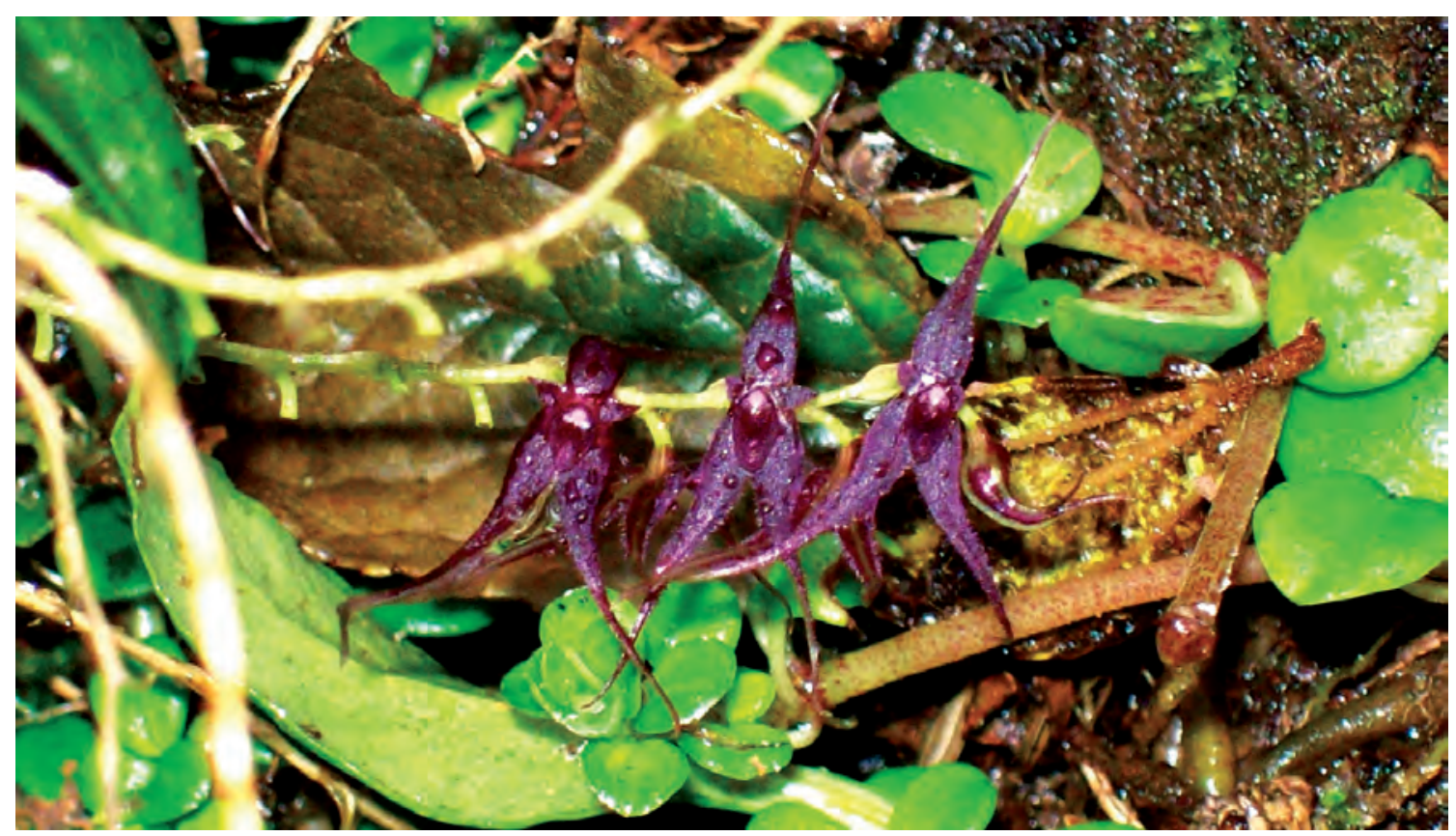

Figura 1. Teagueia moisesii (de M. Chocce et al. 3894). Foto por Miguel Chocce.

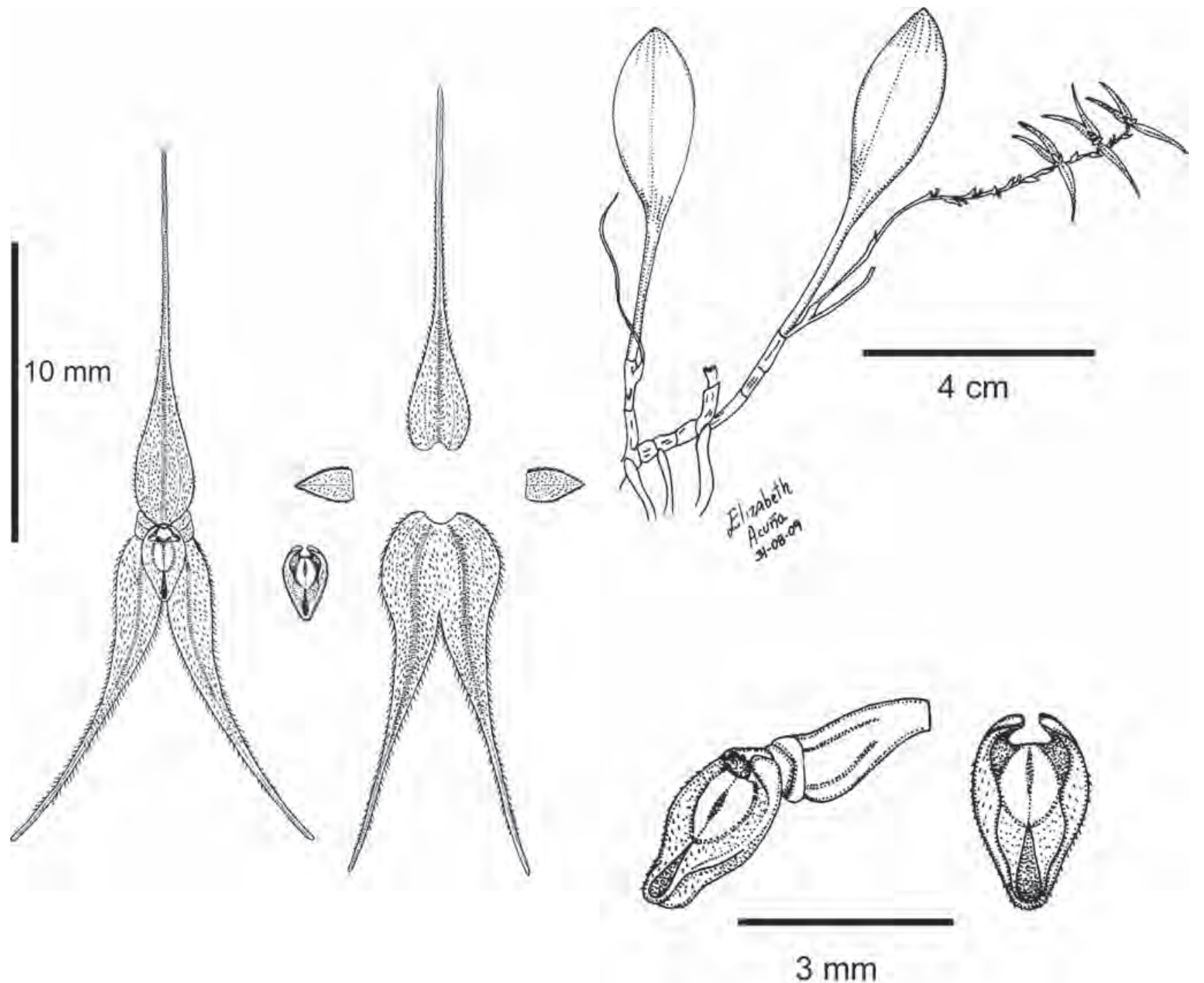

Figura 2. Teagueia moisesii (de M. Chocce et al. 3894). Ilustración por Margoth Acuña-Tarazona 
a la columna; columna cuculada, $1 \mathrm{~mm}$ de largo, casi $1 \mathrm{~mm}$ de ancho, estigma bilobulado (Figs. 1 y 2 ).

Etimología.- En honor a Moisés Miguel Chocce Cosi, padre del primer autor.

Comentarios.- Esta especie se incluye dentro del grupo de especies formado por Teagueia lehmannii Luer, Teagueia phasmida (Luer \& Escobar) Luer, Teagueia rex (Luer \& Escobar) Luer, Teagueia teaguei (Luer) Luer, Teagueia tentaculata Luer \& Hirtz y Teagueia zeus (Luer \& Hirtz) Luer. Es cercano a $T$. phasmida, compartiendo algunas características como las flores totalmente pubescente y la forma del labio, pero se distingue de esta por sus sépalos más pequeños (la mitad del tamaño de T. phasmida), labio ligeramente más pequeño, y las hojas y el ramicaules mucho más grandes (cercano al doble del tamaño de T. phasmida).

\section{Agradecimientos}

Agradecemos al Herbario San Marcos USM, la empresa Candente Resource Corp. que autorizó la publicación de la presente información, obtenida durante el desarrollo de una línea base ambiental en el área de estudio, e INRENA que entregó la autorización para la presente investigación (Resolución Directoral Nº017-2009-AG-DGFFS-DGEFFS). También deseamos agradecer a la Dra. Aída Mendoza Cuba por su ayuda con la diagnosis en latín, a Jorge Lingan por su ayuda con la traducción al inglés y a Sydney Novoa por su ayuda en el trabajo de campo.

\section{Literatura citada}

Jost, L. 2004. Explosive Local Radiation of the Genus Teagueia (Orchidaceae) in the Upper Pastaza Watershed of Ecuador. Lyonia 7: 41-47.

Luer, C.A. 1986. ICONES PLEUROTHALLIDINARUM I: Systematics of the Pleurothallidinae. Monographs in Systematic Botany from the Missouri Botanical Garden vol. 15.

Luer, C.A. 1990. ICONES PLEUROTHALLIDINARUM VII: Systematics of Platystele (Orchidaceae). Monographs in Systematic Botany from the Missouri Botanical Garden vol. 38.

Luer, C.A. 1991. ICONES PLEUROTHALLIDINARUM VIII: Systematics of Lepanthopsis, Octomeria subgenus Pleurothallopsis, Restrepiella, Restrepiopsis, Salpistele, and Teagueia. Monographs in Systematic Botany from the Missouri Botanical Garden vol. 39. 
